\title{
Determination and classification of cutaneous innervation of the dorsum of the foot in foetal cadavers
}

\author{
B. Candan'1, S. Albay ${ }^{2}$ \\ ${ }^{1}$ Department of Anatomy, Faculty of Medicine, Alanya Alaaddin Keykubat University, Alanya, Turkey \\ ${ }^{2}$ Department of Anatomy, Faculty of Medicine, Suleyman Demirel University, Isparta, Turkey
}

[Received: 15 January 2018; Accepted: 11 February 2018]

Background: The aim of this study is to determine cutaneous innervation of the dorsum of the foot on foetal cadavers.

Materials and methods: In this study. 200 limbs from 100 embalmed foetuses (54 males and 46 females) were studied in Anatomy Laboratory. Contributions of medial, lateral and intermediate dorsal cutaneous nerves (MDCN, LDCN and IDCN) of the foot were identified.

Results: Cutaneous innervation of the dorsum of the foot was classified into five types. Type I (75\%) where MDCN innervated medial border of the foot and second interdigital cleft; IDCN innervated third, fourth and fifth interdigital clefts; and LDCN innervated the lateral border of the foot. Type II (21\%) where MDCN innervated medial border of the foot, second and third interdigital clefts; IDCN innervated fourth and fifth interdigital clefts; and LDCN innervated the lateral border of the foot. Type III (1.5\%) where saphenous nerve innervated medial border of the foot; MDCN innervated second and third interdigital clefts; IDCN innervated fourth and fifth interdigital clefts; and LDCN innervated the lateral border of the foot. Type IV (1.5\%) was similar to type I, with an extra connection between the MDCN and IDCN on the dorsum of the foot. Type $V(1 \%)$ where superficial fibular nerve innervated medial border of the foot, and second, third and fourth interdigital clefts; and sural nerve innervated fifth interdigital cleft and lateral border of the foot.

Conclusions: The present study provides a new classification for the cutaneous innervation of the dorsum of the foot. (Folia Morphol. 2018; 77, 4: 698-702)

Key words: foetus, superficial fibular nerve, medial dorsal cutaneous nerve, sural nerve

\section{INTRODUCTION}

The skin of the dorsum of the foot is innervated by the superficial fibular nerve (SFN), deep fibular nerve (DFN) and the sural nerves (SrN). SFN divides into medial dorsal cutaneous nerve (MDCN) and intermediate dorsal cutaneous nerve (IDCN) on the dorsum of the foot $[13,14]$. The MDCN divides into two dorsal digital nerves for the medial side of the great toe and adjacent sides of the second interdigital space. The IDCN innervates the adjacent sides of third to fifth toes through digital branches. SrN continues onto the foot as the lateral dorsal cutaneous nerve (LDCN) supplying lateral aspect of the little toe. Skin of first interdigital cleft is supplied by DFN [11]. Anatomical 
variations in the course and distribution of cutaneous nerves are important in planning surgical interventions around leg, ankle and foot [9]. Knowledge of the variability of peripheral nerve distribution has utmost clinical importance [7]. It is well-known that cutaneous branches in the ankle and foot regions are frequently at risk of injury during various operative procedures [14].

Variations in the pattern of cutaneous innervation have been reported in an early study in 1926 [5], another one in 1987 [15] and also in more recent studies $[2,7,10,12]$. A few studies on human foetuses $[3,6]$ describe the course and branching pattern of SFN in the leg. The detailed branching pattern of the SFN in foetuses may be important in tumour and deformity surgery in the early period of life. Hence, the aim of our study was to classify the different patterns of cutaneous innervation on the dorsum of the foot in Turkish foetuses.

\section{MATERIALS AND METHODS}

The study was carried out on 200 lower limbs of 54 male and 46 female foetuses, ranging between 19 and 40 weeks of gestation. Foetuses with no external pathology or anomaly were obtained from Isparta Maternity and Children's Hospital. The foetuses were embalmed in 10\% formalin and dissections were performed in the Department of Anatomy. Written consents from the families and approval from the Ethics Board of the Suleyman Demirel University Faculty of Medicine (11/19/2014, No. 182) were obtained at the beginning of the study, prior to foetal dissections. Gestational ages between the $13^{\text {th }}$ and $40^{\text {th }}$ weeks were determined by biparietal diameter, head circumference, femur length and foot length. Foetuses were divided into the following three groups according to their gestational ages: 13-25 weeks, second trimester (52 lower limbs, 28 male, 24 female); 26-37 weeks, third trimester (98 lower limbs, 52 male, 46 female) and 38-40 weeks, full term (50 lower limbs, 28 male, 22 female). A longitudinal skin incision was made from lateral epicondyle to the lateral malleolus, and an additional skin incision was made from the ankle to the median digit of the foot. Furthermore, a horizontal skin incision was made between the lateral malleolus and the medial malleolus. After careful reflection of the skin, the nerves of the dorsum of the foot could be easily seen. Contributions of MDCN, LDCN and IDCN of the foot were identified.

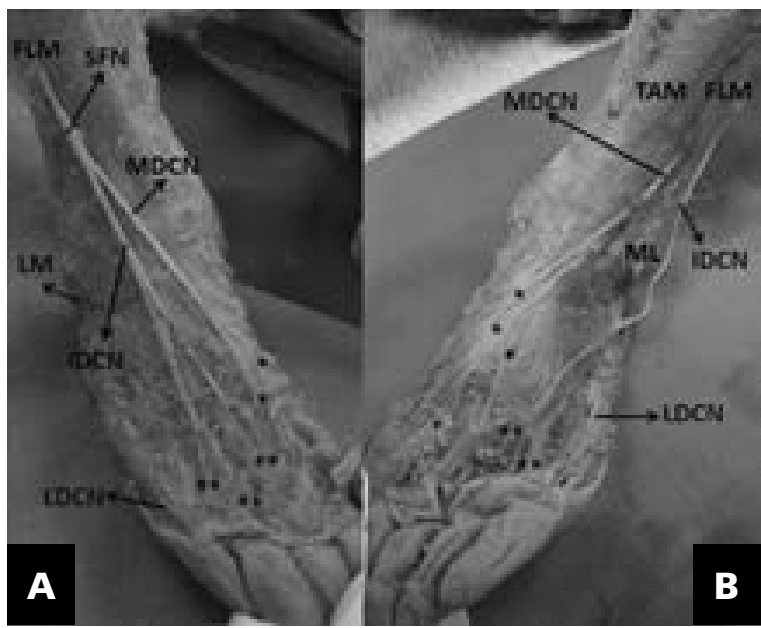

Figure 1. A. Type I; B. Type Il; *Branch of MDCN; **Banch of IDCN; FLM — fibularis longus muscle; IDCN — intermediate dorsal cutaneous nerve; LDCN — lateral dorsal cutaneous nerve; LM lateral malleolus; MDCN - medial dorsal cutaneous nerve; SFN superficial fibular nerve; TAM — tibialis anterior muscle.

\section{RESULTS}

The contributions of MDCN, IDCN and LDCN to cutaneous innervation of the foot were evaluated. Cutaneous innervation of the dorsum of the foot was classified into five types. The most common pattern was type I ( $75 \%, 150$ of 200 cases), where SFN divided into MDCN and IDCN near the ankle, and MDCN innervated the medial border of great toe and adjacent sides of second interdigital cleft. IDCN supplied third, fourth and fifth interdigital clefts. The SrN through its continuation as LDCN supplied lateral border of foot (Fig. 1A). In type II ( $21 \%, 42$ of 200 cases), MDCN and IDCN pierced the deep fascia of the leg independently in the lower leg; MDCN innervated medial border of the foot, second and third interdigital clefts; IDCN innervated fourth and fifth interdigital clefts; and LDCN innervated the lateral border of the foot (Fig. 1B). In type III (1.5\%, 3 of 200 cases) saphenous nerve (SN) innervated medial border of the foot, MDCN innervated second and third interdigital clefts, IDCN innervated fourth and fifth interdigital clefts, and LDCN innervated the lateral border of the foot (Fig. 2A, B). Type IV (1.5\%, 3 of 200 cases) was similar to type I, as MDCN innervated the medial border of the foot and second interdigital cleft, IDCN innervated third, fourth and fifth interdigital clefts, and LDCN innervated the lateral border of the foot; however, in type IV there was a connection between the MDCN and IDCN at the dorsum of the foot (Fig. 3A). In type $V(1 \%, 2$ of 200 cases) SFN innervated medial side of the foot, 


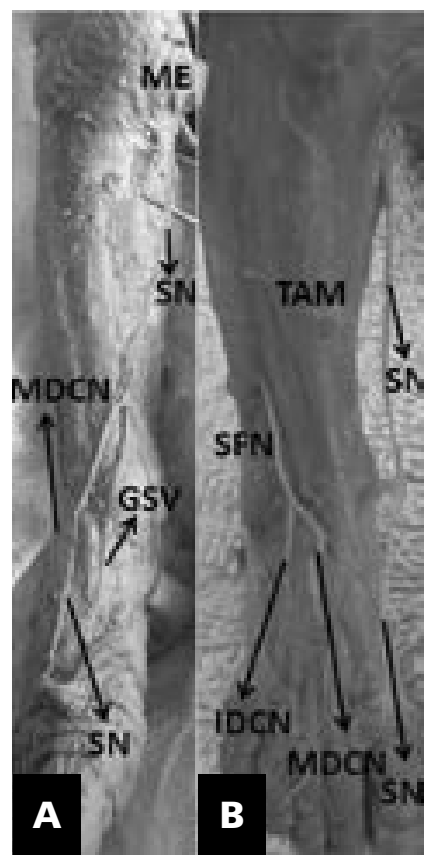

Figure 2. A, B. Contribution of the saphenous nerve to the dorsum of the foot in type III; GSV - great saphenous vein; IDCN - intermediate dorsal cutaneous nerve; MDCN - medial dorsal cutaneous nerve; $\mathrm{ME}$ — medial epicondyle; SFN — superficial fibular nerve; SN — saphenous nerve; TAM — tibialis anterior muscle.

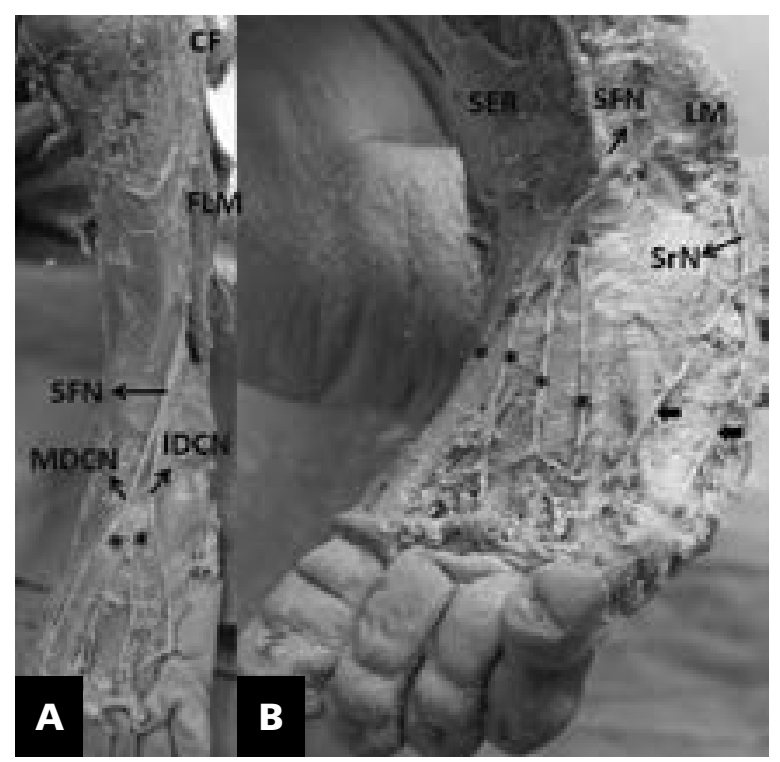

Figure 3. A. Type IV, connection between MDCN and IDCN; B. Type V; black arrow - branch of sural nerve; ${ }^{*}$ In panel $A$ indicates the communicating branch; *In panel B indicates the branch of SFN; CF — caput fibulae; FLM — fibularis longus muscle; IDCN intermediate dorsal cutaneous nerve; LM — lateral malleolus; MDCN - medial dorsal cutaneous nerve; SER — superior extensor retinaculum; SFN — superficial fibular nerve; SrN — sural nerve.

second, third and fourth interdigital clefts, and SrN innervated fifth interdigital cleft and lateral border of the foot (Fig. 3B).
The first interdigital cleft was supplied by DFN in all of types.

\section{DISCUSSION}

Although cutaneous innervation of the dorsum of the foot has been reported in many studies on adult $[4,7,12]$, limited number of studies are available on foetuses $[14,15]$. In other studies, authors classified the cutaneous innervation of the dorsum of the foot according to the distribution of MDCN, IDCN and LDCN, and they considered the connecting branches between these cutaneous nerves as subgroups. In our study, we classified the cutaneous innervation of the dorsum of the foot into five types, according to the distribution of MDCN, IDCN, LDCN, and connecting branches between these cutaneous nerves (Fig. 4). The most common presentation in our study, the type I (75\%), has been frequently described in textbooks of anatomy. It was also the most common type described in Indian foetuses (41.6\%) and Indian population $(35.4 \%)[7,14]$. However, this type was described in $17.6 \%$ of the Australian [12] and $13.3 \%$ of the Turkish populations [4] and in $13.75 \%$ of the Polish foetuses [15]. Type II was the second common type in this study (21\%); it was described in 3.3\% of Indian foetuses by Wahee et al. [14] and they classified this type as type $1 \mathrm{~b}$. This type was described in $17.6 \%$ and $35.4 \%$ of the Australian and Indian populations, respectively $[7,12]$. These rates are consistent with our findings. In type III (1.5\%) we found that SN innervated the medial border of the foot. Ziótkowski et al. [15] found this ratio $1.88 \%$ in 160 extremities of 80 foetuses. In the absence of the MDCN, the SN innervates the medial part of the foot $(0.8 \%)$ [6]. These rates are consistent with our rate. In type IV (1.5\%) we identified a communication between a branch of MDCN (second interdigital cleft) and a branch of IDCN (third interdigital cleft). The frequency of this communication has been reported as $4 \%$ [1], 5\% [7], 8.3\% [14], 24.9\% [5] in the literature. In type $V(1 \%)$ we identified an undivided SFN continued over the medial border of the foot, second, third and fourth interdigital clefts, and SrN innervated fifth interdigital cleft and lateral border of the foot. While in the absence of the IDCN, the SrN supplies the lateral part of the dorsum of the foot [1]. This type was described in $18.3 \%$ of Indian foetuses by Wahee et al. [14], and they considered this type as type $2 \mathrm{a}$. The frequency of this type was reported as $26.5 \%$ [12], $24.6 \%$ [7], $10 \%$ [4] and $2.5 \%$ [6] 


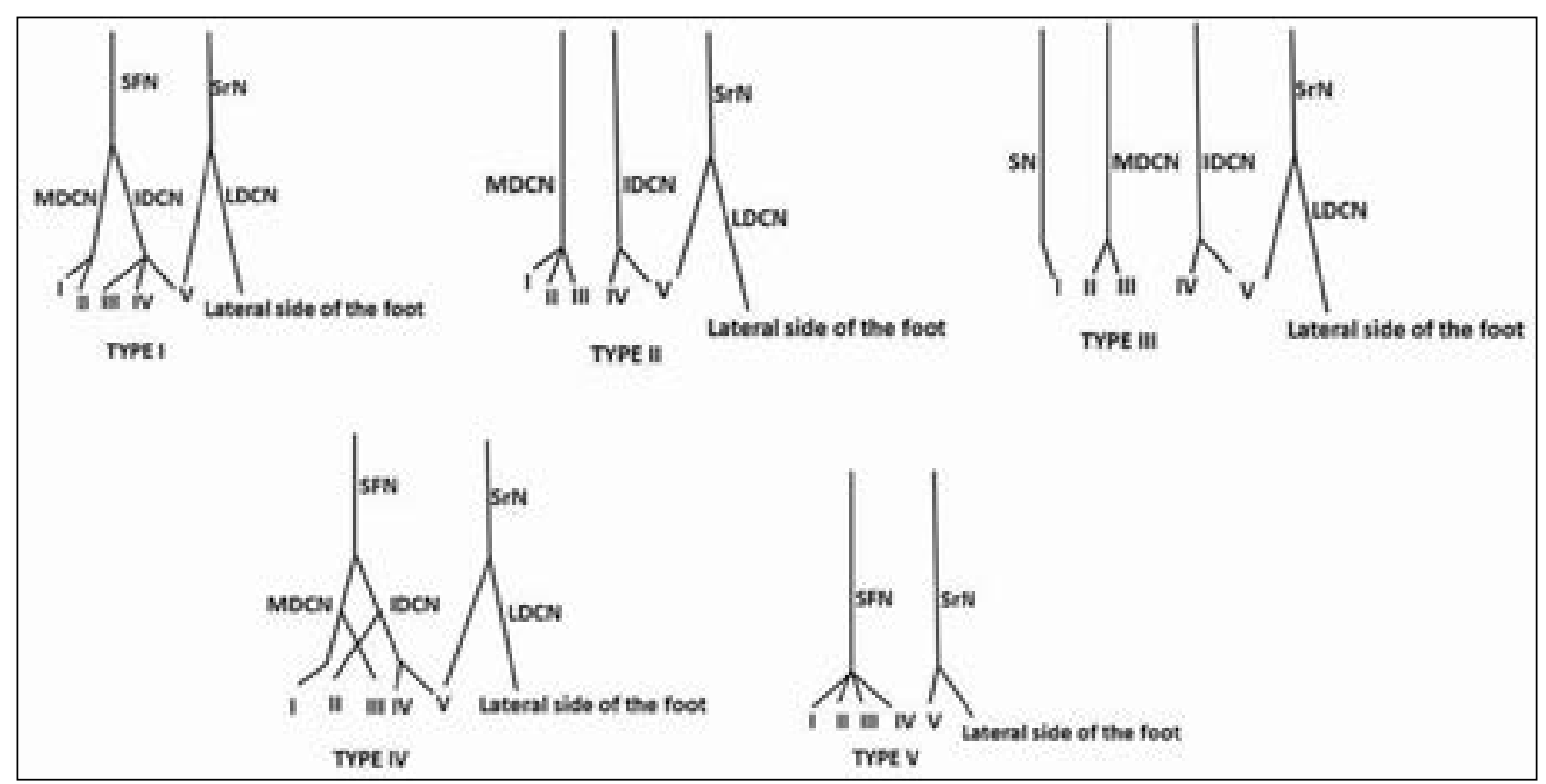

Figure 4. Schematic drawings of the typology of the cutaneous nerves; I-II-III-IV-V — represent fingers; IDCN — intermediate dorsal cutaneous nerve; LDCN — lateral dorsal cutaneous nerve; MDCN — medial dorsal cutaneous nerve; SFN — superficial fibular nerve; $\mathrm{SN}$ - saphenous nerve; SrN — sural nerve.

in the literature. Our rate is consistent with the rate of this type in Kurtoglu et al.'s study in Turkish population [6].

Knowledge about variations in cutaneous innervation patterns on the dorsum of the foot improves understanding of the variability of distal sensory loss in nerve entrapments and iatrogenic nerve injury [14]. It is known that sensory branches like MDCN, IDCN and $\mathrm{SrN}$ in the region of ankle and foot, are frequently at risk of injury during various operative procedures in the region [1, 12]. Also, terminal branch of SFN in the supramalleolar region or the dorsum of the foot has a role in flap surgery $[4,8]$.

\section{CONCLUSIONS}

The cutaneous innervation on the dorsum of the foot in Turkish foetuses was classified as five main types. A comparison with other cutaneous innervation types in other populations revealed many differences in frequency. A new classification is described in the present work. The results of our study revealed differences in distribution pattern of the cutaneous innervation of the foot in foetuses. We hope that our study might be useful in future studies.

\section{Acknowledgements}

We declare that study design, data collection, data analysis and manuscript preparation were carried out by the authors. We thank the families of foetuses for their contributions to this study.

\section{REFERENCES}

1. Blair JM, Botte MJ. Surgical anatomy of the superficial peroneal nerve in the ankle and foot. Clin Orthop Relat Res. 1994(305): 229-238, indexed in Pubmed: 8050234.

2. Büyükmumcu $M$, Ustün $M E$, Seker $M$, et al. The possibility of deep peroneal nerve neurotisation by the superficial peroneal nerve: an anatomical approach. J Anat. 1999; 194 ( Pt 2): 309-312, indexed in Pubmed: 10337964.

3. Domagała Z, Gworys B, Porwolik K. Preliminary assessment of anatomical variability of nervus peroneus superficialis in the foetal period. Folia Morphol. 2003; 62(4): 401-403, indexed in Pubmed: 14655126.

4. Aktan Ikiz ZA, Ucerler H. The distribution of the superficial peroneal nerve on the dorsum of the foot and its clinical importance in flap surgery. Foot Ankle Int. 2006; 27(6): 438-444, doi: 10.1177/107110070602700609, indexed in Pubmed: 28895480.

5. Kosinski $C$. The course, mutual relations and distribution of the cutaneous nerves of the metazonal region of leg and foot. J Anat Physiol. 1926; 60(Pt 3): 274-297, indexed in Pubmed: 17104102.

6. Kurtoglu Z, Aktekin M, Uluutku MH. Branching patterns of the common and superficial fibular nerves in fetus. Clin Anat. 2006; 19(7): 621-626, doi: 10.1002/ca.20235, indexed in Pubmed: 16302233.

7. Madhavi C, Isaac B, Antoniswamy B, et al. Anatomical variations of the cutaneous innervation patterns of the sural nerve on the dorsum of the foot. Clin Anat. 2005; 18(3): 206-209, doi: 10.1002/ca.20094, indexed in Pubmed: 15768411. 
8. Malikov S, Casanova D, Magualon G, et al. Surgical anatomy of the lateral supramalleolar flap in arteritic patients: an anatomic study of 24 amputation specimens. Surg Radiol Anat. 2003; 25(2): 89-94, doi: 10.1007/s00276003-0098-x, indexed in Pubmed: 12715208.

9. Prakash, Bhardwaj AK, Singh DK, et al. Anatomic variations of superficial peroneal nerve: clinical implications of a cadaver study. Ital J Anat Embryol. 2010; 115(3): 223-228, indexed in Pubmed: 21287977.

10. Rubel IF, Schwarzbard I, Leonard A, et al. Anatomic location of the peroneal nerve at the level of the proximal aspect of the tibia: Gerdy's safe zone. J Bone Joint Surg Am. 2004; 86-A(8): 1625-1628, indexed in Pubmed: 15292408.

11. Ryan W, Mahony N, Delaney M, et al. Relationship of the common peroneal nerve and its branches to the head and neck of the fibula. Clin Anat. 2003; 16(6): 501-505, doi: 10.1002/ca.10155, indexed in Pubmed: 14566896.

12. Solomon LB, Ferris $L$, Tedman R, et al. Surgical anatomy of the sural and superficial fibular nerves with an emphasis on the approach to the lateral malleolus. J Anat. 2001; 199(Pt 6): 717-723, indexed in Pubmed: 11787825.

13. Standring S. Leg. In: Standring S (ed) Gray's anatomy. The anatomical basis of clinical practice 39th edn. Elsevier Churchill Livingstone, Edinburgh 2005: 1504-1505.

14. Wahee P, Aggarwal A, Sahni D. Variable patterns of cutaneous innervation on the dorsum of foot in fetuses. Surg Radiol Anat. 2010; 32(5): 469-475, doi: 10.1007/s00276009-0587-7, indexed in Pubmed: 19911110.

15. Ziółkowski M, Suder E, Porwolik K, et al. Skin innervation of the foot in human fetuses. Folia Morphol. 1987; 46(1-2): 57-65, indexed in Pubmed: 3507332. 